\title{
Seroprevalencia de infección por Leptospira y factores de riesgo en estudiantes de una universidad de Colombia
}

\section{Seroprevalence of Leptospiral infection and risk factors in students of a Colombian university}

Título abreviado: Seroprevalencia de infección por Leptospira

Sandra Helena Suescún Carrero ${ }^{1}$, Dina Paola HerediaMontoya ${ }^{2}$, Yoryany Mulato Bolaños², Martín Orlando Pulido Medellín ${ }^{3}$

\section{Resumen}

Objetivo. Determinar la seroprevalencia de Leptospira spp y factores de riesgo en estudiantes de medicina veterinaria y zootecnia de la Universidad de Tunja, Colombia. Método. Se utilizó la prueba de microaglutinación (MAT) para 13 serovares de Leptospira en sueros de 51 estudiantes. Los datos epidemiológicos y factores de riesgo, se recogieron a través de una encuesta. Resultados. La seroprevalencia de Leptospira fue 25,5\% (IC95\% 13,1 - 36,8). La edad promedio de los estudiantes fue 26,2 años; $\mathrm{DE}=4,15)$. La prevalencia fue mayor en hombres que en mujeres $(36,6 \%$ vs. $20 \%, p>0,05)$ sin diferencia estadística. La reactividad a los serovares de Leptospira fue 5,88\% para Australis (serovar bratislava), Grippotyphosa (serovar Grippotyphosa), Sejroe (serovar Hardjo Prajitno), Pomona (serovar Pomona) y Tarassovi (serovar Tarassovi); y de 1.96\% para Canicola (serovar Canicola), Icterohaemorragiae (serovar Copenhageni) y Shermani (serovar Shermani). Se encontró que trabajar o tener práctica clínica durante los seis meses anteriores a la toma de la muestra representó un riesgo de $2,25(0,1209-2,1342)$ veces para presentar leptospirosis y el haber tenido accidentes laborales en el desarrollo de las actividades como salidas de campo o en la práctica clínica, representó 2,69 $(0,6393$ - 11,3892) veces el riesgo de presentar seropositividad. Las variables de riesgo asociadas no fueron significativas $(p=0,05)$. Conclusión. De acuerdo con los resultados se puede observar que la Leptospirosis es una enfermedad de gran importancia y de elevada seropositividad en el departamento de Boyacá, principalmente en la población que se encuentra constantemente en riesgo ocupacional al trabajar con animales.

Palabras claves: Leptospirosis, infección, factores de riesgo,estudios seroepidemiológicos, estudiantes, Colombia.

1. Bacterióloga, Magister en Administración en Salud, Grupo de Investigación Hygea. Universidad de Boyacá. Tunja, Colombia.
2. Bacterióloga y Laboratorista clínica, Universidad de Boyacá Tunja, Colombia.
3. Médico Veterinario, Maestría en Ciencias Biológicas, Grupo de Investigación GIDIMEVETZ, Programa de MVZ, Universidad Pedagógica y
Tecnológica de Colombia, Tunja, Colombia. 


\begin{abstract}
Objective. To determine the seroprevalence of Leptospira spp and risk factors in medicine veterinary' students of a University in Tunja, Colombia. Method. The microscopic agglutination test was used with 13 leptospiral serovars in sera of 51 students. Epidemiological data and risk factors were collected through a survey. Results. Leptospira seroprevalence was 25.5\% (IC95\% 13,1 - 36,8). The average age of students was 26, 2 years; $\mathrm{DE}=4.15)$. The prevalence was higher in men than in women $(36.6 \%$ vs. $20 \%, p=0.1306)$, being statistically the same. Reactivity to Leptospira serovars was 5.88\% for Australis (serovar bratislava), Grippotyphosa (serovar Grippotyphosa), Sejroe (serovar Hardjo Prajitno), Pomona (serovar Pomona) y Tarassovi (serovar Tarassovi); and of 1.96\% for Canicola (serovar Canicola), Icterohaemorragiae (serovar Copenhageni) y Shermani (serovar Shermani). It was found that working or having clinical practice during the six months prior to sampling represented $2.25(0.1209$ - 2.1342) times the risk for getting leptospirosis; and have had work accidents in the development of activities as field trips or in clinical practice represented $2.69(0.6393$ - 11.3892) times the risk for getting leptospirosis. Risk variables associated with seroprevalence were not significant $(p=0.05)$. Conclusion. According to the results, it is evident that the Leptospirosis is a disease of great importance and high recurrence in the department of Boyacá, mainly among the population working with animals, which is in constant occupational risk.
\end{abstract}

Keywords: Leptospirosis, Infection, Risk Factors, Seroepidemiologic Studies, Students, Colombia.

\section{Introducción}

La leptospirosis es una enfermedad zoonótica de distribución mundial, que afecta a los humanos en todos los continentes, tanto en contextos urbanos como rurales y en climas templados y tropicales (1). Los animales son considerados hospederos de mantenimiento de la enfermedad (2) y los distintos serovares tienen predilección por algunas especies (3), entre los que nombran: L. canicola asociada a caninos, L. hardjo para bovinos, L. pomona para bovinos y porcinos, $L$. bratislava para porcinos (4) y L. icterohaemorrhagiae a roedores y caninos, siendo ésta la que produce con mayor frecuencia infección grave en humanos (5). La fuente de contagio es la orina de animales enfermos o reservorios, la forma de transmisión puede ser directa mediante el contacto con orina infectada, fluidos fetales y placentarios o descargas uterinas, y puede ser indirecto por contacto con ambiente (agua o suelos) o alimentos contaminados (6). La vía de ingreso se da a través de la piel lacerada o mucosas (oral, nasal o conjuntiva), e incluso a través de la piel intacta después de una prolongada inmersión en el agua; también puede ser por ingestión o inhalación (7). De acuerdo con lo reportado por la OMS, el número de casos de leptospirosis en humanos en el mundo no ha sido completamente documentado, dado que en varios países no se dispone de sistemas de vigilancia y estadísticas para esta enfermedad, y en varias zonas esta no es correctamente diagnosticada o los casos no son reportados como leptospirosis, debido a que otras enfermedades presentan signología similar. La OMS ha establecido una probabilidad de 1 caso por cada 100.000 habitantes por año en climas templados y 10 casos por cada 100.000 habitantes por año en climas tropicales húmedos (8). La incidencia de leptospirosis en América se ha estimado en 12.5 casos por 100.00 habitantes, y se reportan casos de leptospirosis principalmente en Brasil (9).

Brasil reporta datos epidemiológicos con una incidencia anual de 1,9/100.000 habitantes, con ocurrencia en todos los meses del año y con una letalidad media de 9\%. (10). En México, se reporta una 
tasa de 0.65 en el 2000 y al 2010 de 0.45 casos por cada 100,000 habitantes, manteniéndose constante durante los últimos 10 ańos (11). En Argentina, según el Sistema Nacional de Vigilancia de la Salud, hasta la semana epidemiológica 46 se notificaron 1287 casos y se confirmaron 133 casos en el 2014 (12). En Chile, durante el período 2001-2009 se notificaron a través del sistema de vigilancia epidemiológica de enfermedades obligatorias 137 casos (13). Y en Perú, en un estudio realizado en estudiantes de medicina veterinaria al inicio y término de la carrera se encontró una seropositividad de $18.4 \%$ y de $8.8 \%$ respectivamente (14).

De acuerdo con la información del Sistema Nacional de Vigilancia en Salud Pública (Sivigila), hasta la semana epidemiológica 16 de 2015 se han reportado 771 casos de leptospirosis en Colombia, frente a 753 en la misma semana del año anterior, lo que implica un incremento en la notificación de 2,33 $\%$. Los casos proceden de 33 entidades territoriales, el 59,58 \% de los reportes se concentraron en Valle del Cauca, Antioquia y Tolima. La proporción de incidencia nacional acumulada hasta la semana epidemiológica 16 es de 0,324 casos por cada 100.000 habitantes, las entidades territoriales de Guaviare, Amazonas, Chocó y Arauca son las que presentan la mayor incidencia en el país (15). El objetivo de este estudio fue determinar la seroprevalencia de Leptospira spp y los factores de riesgo en estudiantes de Medicina Veterinaria y Zootecnia de una Universidad de Tunja, Colombia.

\section{Materiales y métodos}

Área de estudio. Se realizó un estudio observacional, descriptivo y transversal. La población de estudio estuvo conformada por 51 estudiantes de medicina veterinaria y zootecnia de octavo, noveno y décimo semestre que corresponden al total (100\%) de los estudiantes que se encontraban realizando las prácticas clínicas en el segundo semestre del año 2014. Los datos epidemiológicos y los posibles factores de riesgo asociados con Leptospira sp, se recogieron a través de una encuesta realizada a cada participante.
Toma de muestras. Se obtuvo una muestra de $5 \mathrm{ml}$ de sangre venosa. Las muestras fueron tomadas en tubo Vacutainer ${ }^{\circledR}$ sin anticoagulante, se dejaron coagular y se centrifugaron a 2.500rpm durante cinco minutos. Se separó el suero en viales, los cuales fueron conservados a $-20^{\circ} \mathrm{C}$ durante 15 días para analizarlos mediante la prueba de microaglutinación MAT.

Prueba de microaglutinación (MAT). Mediante un panel de 13 serovares de Leptospira como antígenos vivo, se aplicó la técnica de MAT. Estas cepas pertenecían a 13 serogrupos: Autumnalis (serovar autumnalis), Bataviae (serovar bataviae), Australis (serovar bratislava), Canicola (serovar canicola), Icterohaemorragiae (serovar copenhageni), Cynopteri (serovar cynopteri), Grippotyphosa (serovar grippotyphosa), Sejroe (serovar hardjo prajitno), Mini (serovar mini), Pomona (serovar pomona), Shermani (serovar shermani), Tarassovi (serovar tarassovi), Semaranga (serovar Semaranga). Las leptospiras usadas como antígenos fueron cultivadas en medio líquido (Leptospira medio base EMJH) y se ajustaron al estándar de la escala de McFarland de 0,5. La prueba se realizó en microplacas de 96 pocillos agregando $8 \mu \mathrm{l}$ suero más $92 \mu \mathrm{l}$ PBS (1/12.5); a partir de esta dilución se hacen diluciones seriadas con $50 \mu \mathrm{l}$ PBS y $50 \mu \mathrm{l}$ de la primera dilución; posteriormente se procede a adicionar a las diluciones $50 \mu \mathrm{l}$ de cultivo de Leptospiras diluido (1:cultivo/3 diluyente PBS). Las placas se cubrieron e incubaron durante 1 hora a 37 ${ }^{\circ} \mathrm{C}$. La prueba se interpretó como positiva al observar al microscopio de campo oscuro con objetivo de 10x aglutinación igual o mayor al 50\% de las Leptospiras con al menos uno de los serovares y en una dilución del suero de 1:50, a los sueros positivos se les realizaron diluciones seriadas. En cada uno de los procesamientos se montó un control positivo, uno negativo y un control de antígenos.

Análisis estadístico. Para el análisis univariado de datos, se establecieron frecuencias y porcentajes; para el análisis bivariado se practicó la prueba de chi cuadrado o la prueba exacta de Fisher según el caso, considerando un $p<0,05$ como significativo, así 
como la razón de momios odds ratios (OR), con su intervalo de confianza del $95 \%$, para establecer asociación entre la presencia de anticuerpos y los factores de riesgo potencialmente asociados. Se efectuó mediante el paquete estadístico SPSS ${ }^{\oplus}$ versión 22.

Aspectos éticos. El estudio fue aprobado por el Comité de Ética de la Universidad de Boyacá, se clasificó como un estudio de riesgo mínimo. La recolección de las muestras y la obtención de la información se ajustaron a las normas éticas de garantía de la confidencialidad, de los beneficios y del riesgo mínimo para los participantes, quienes firmaron el consentimiento informado antes de su participación en el estudio, de acuerdo con lo dispuesto en la Resolución 8430 de 1993, expedida por el Ministerio de Salud y Protección Social de Colombia.

\section{Resultados}

Se obtuvo un total de 51 muestras de suero de estudiantes de Medicina Veterinaria y Zootecnia de las áreas prácticas, de las cuales 25,5\% (IC95\% 13,1 $36,8)$ fueron positivas para al menos un serovar. La edad promedio de los estudiantes fue de 26,2 años; $\mathrm{DE}=4,15$. La prevalencia fue mayor en hombres que en mujeres $(36,6 \%$ vs. $20 \%, p=>0,05)$

En cuanto a los posibles factores de riesgo relacionados con la presencia de anticuerpos anti-Leptospira, el 78,4\% manifestó convivir con animales domésticos principalmente perros, gatos, caballos y vacas; el 86,2\% trabaja o tiene práctica clínica durante los seis meses anteriores a la toma de la muestra. Esta condición representó 2,25 ( 0,1209-2,1342) veces el riesgo de tener leptospirosis, sin asociación estadísticamente significativa $(0,418)$; el $80 \%$ ha tenido contacto directo con suelo; el 53\% manifestó haber estado expuesto a diferentes fuentes de agua (arroyo, lagos, lagunas, aguas estancadas, pozos o manantiales) durante sus prácticas; un bajo porcentaje de estudiantes 17,4\% manifestó caminar descalzo. Por el contrario, el 60,8\% contestó que ha tenido accidentes laborales en el desarrollo de sus actividades ya sea en las salidas de campo o en la clínica de grandes y pequeños animales de la universidad, dichas lesiones corresponden a pinchazos con agujas, cortaduras o abrasiones, esta condición representó 2,69 (0,6393 - 11,3892) veces el riesgo de tener leptospirosis. El $48 \%$ manifestó consumir productos no pasteurizados, la mayoría de estudiantes cumplían con las normas de bioseguridad durante las prácticas como uso de guantes $98 \%$; uso de gorro $80 \%$; uso de tapabocas $90 \%$ y uso de bata $86 \%$, presentándose esta condición como un factor protector para leptospirosis. No se encontró asociación estadísticamente significativa al analizar las anteriores variables entre el grupo de personas seropositivas y seronegativas. Tabla 1. 
Tabla 1. Factores relacionados con la presencia de anticuerpos anti-Leptospira en la población estudiada.

\begin{tabular}{|c|c|c|c|c|}
\hline Variable & Positivos n $\quad(\%)$ & Negativos $\mathbf{n} \quad(\%)$ & OR (IC95\%) & Valor $p$ \\
\hline & $\mathrm{n}=13$ & $\mathrm{n}=38$ & & \\
\hline Sí & $9(17,6 \%)$ & $31(60,8 \%)$ & 0,5081 & \multirow[t]{2}{*}{ ** 0.2850511} \\
\hline No & $4(7,8 \%)$ & $7(13,7 \%)$ & $(0,1209-2,1342)$ & \\
\hline Sí & $12(23,5 \%)$ & $32(62,7 \%)$ & 2,25 & \multirow{2}{*}{${ }^{* *} 0.41899432$} \\
\hline No & $1(2,0 \%)$ & $6(11,8 \%)$ & $(0,2447-20,6869)$ & \\
\hline \multicolumn{4}{|l|}{ Contacto directo con suelo } & ** 0.5154918 \\
\hline \multicolumn{4}{|l|}{ Contacto directo u oral con agua } & \multirow{3}{*}{${ }^{*} 0.79638907$} \\
\hline $\mathrm{Si}$ & $6(12,2 \%)$ & $20(40,8 \%)$ & 0,6857 & \\
\hline No & $7(14,3 \%)$ & $16(32,7 \%)$ & $(0,1919-2,4498)$ & \\
\hline \multicolumn{4}{|l|}{ Camina descalzo } & \multirow{3}{*}{${ }^{* *} 0.6$} \\
\hline Sí & $1(2,2 \%)$ & $7(15,2 \%)$ & 0,3095 & \\
\hline No & $12(26,1 \%)$ & $26(56,5 \%)$ & $(0,0342-2,8051)$ & \\
\hline \multicolumn{4}{|l|}{ Consume productos no pasteurizados } & ${ }^{*} 0.261482654$ \\
\hline \multicolumn{4}{|l|}{ Uso de gorro } & \multirow{3}{*}{${ }^{* *} 0,0670299$} \\
\hline Sí & $8(16,0 \%)$ & $32(64,0 \%)$ & 0,25 & \\
\hline No & $5(10,0 \%)$ & $5(10,0 \%)$ & $(0,058-1,0784)$ & \\
\hline \multicolumn{4}{|l|}{ Uso de tapabocas } & \multirow{3}{*}{${ }^{* *} 0,6109621$} \\
\hline Sí & $12(24,0 \%)$ & $33(66,0 \%)$ & 1,4545 & \\
\hline No & $1(2,0 \%)$ & $4(8,0 \%)$ & $(0,1475-14,3461)$ & \\
\hline \multicolumn{4}{|l|}{ Uso de bata } & \multirow{3}{*}{${ }^{* *} 0,2539613$} \\
\hline Sí & $10(20,0 \%)$ & $33(66,0 \%)$ & 0,404 & \\
\hline No & $3(6,0 \%)$ & $4(8,0 \%)$ & $(0,0772-2,1160)$ & \\
\hline \multicolumn{4}{|l|}{ Herida o accidente trabajo } & \multirow{3}{*}{${ }^{*} 0.29294748$} \\
\hline Sí & $10(19,6 \%)$ & $21(41,2 \%)$ & 2,6984 & \\
\hline No & $3(5,9 \%)$ & $17(33,3 \%)$ & $(0,6393-11,3892)$ & \\
\hline & & $*^{*}$ chi cuadrado $\left(\mathrm{xi}^{2}\right)$ & ${ }^{* *}$ Fisher & nificativo $<0.05$ \\
\hline
\end{tabular}

Fuente. Autores. 
Del panel de los 13 serogrupos evaluados 8 fueron reactivos. Se encontró reactividad frente a los diferentes serovares de Leptospira: Australis (serovar bratislava), Grippotyphosa (serovar Grippotyphosa), Sejroe (serovar Hardjo Prajitno), Pomona (serovar Pomona, Tarassovi (serovar Tarassovi), Canicola (serovar Canicola), Icterohaemorragiae (serovar Copenhageni) y Shermani (serovar Shermani). Figura 1. No se detectaron anticuerpos contra cinco serovares: Autumnalis (serovar autumnalis), Bataviae (serovar bataviae), Cynopteri (serovar cynopteri), Mini (serovar mini) y Semaranga (serovar Semaranga. De las 51 muestras 9 (17\%) fueron positivas para un serovar, 3 (6\%) muestras fueron positivas para dos serovares y una $(2 \%)$ fue positiva para tres serovares de Leptospira spp.

Figura 1. Distribución de la reactividad para Leptospira por serogrupos en estudiantes de medicina veterinaria y zootecnia.

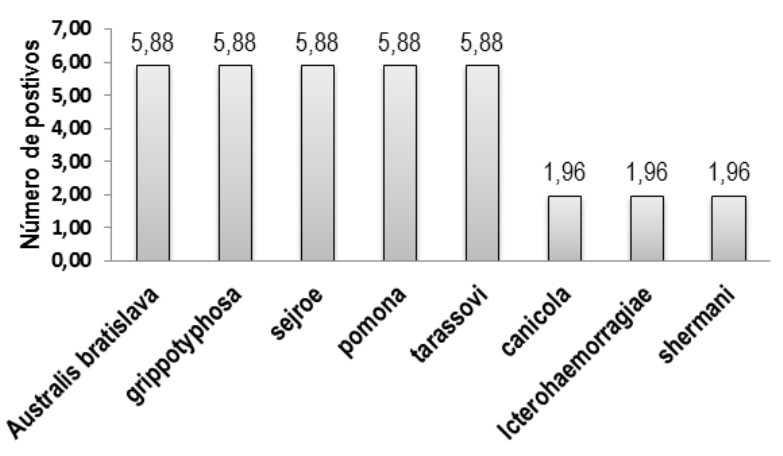

Fuente. Autores

\section{Discusión}

La prevalencia de anticuerpos anti Leptospira encontrada fue del $25,5 \%$, resultado similar a otros estudios realizados, como en el departamento de Boyacá, donde se encontró una prevalencia de $21,7 \%$ en propietarios de caninos de la ciudad de Tunja, donde juega un papel importante el contacto con caninos (16). En otras zonas de Colombia, se encontró en algunos barrios de Cali una prevalencia de 23,3\% (17) y en Villavicencio de 17\% (18). Otro estudio que se llevó a cabo con trabajadores de plantas de beneficio del departamento de Boyacá, reportó una prevalencia mayor 35\%, que la encontrada en el presente estudio (19). En estudios desarrollados en diferentes partes del mundo se han reportado prevalencias inferiores como en India 4,3\%, Malasia $8,4 \%$ y Perú $11,8 \%(20-21,14)$. Estos estudios demuestran prevalencias principalmente en personas expuestas entre los diferentes vectores y hospederos, y una elevada tasa de diseminación entre las personas que presentan alto riesgo laboral por su constante contacto con soluciones de continuidad y sangre de animales contaminados.

Los serovares que mayor seroprevalencia presentaron en este estudio fueron L. bratislava, L. grippotyphosa, L. hardjo, L. tarassovi, L. pomona y $L$ canicola, datos que coinciden con lo reportado en estudios realizados en Turbo, Colombia (22) y en el estado de Yucatán, México (23). Sin embargo, se encontró que los resultados obtenidos en estudiantes de medicina veterinaria y zootecnia del presente estudio, difieren del estudio realizado en Villavicencio, Colombia, donde la mayor seroprevalencia se obtuvo en los serovares L. javanica, L. wollfi, y no se encontró prevalencia de los serovares $L$. tarassovi, $L$. hardjo y L. bratislava (24). Se encontró una diferencia significativa en la prevalencia de infección entre hombres y mujeres, siendo mayor en los hombres, hallazgos que coinciden con lo reportado en otros estudios $(20,25)$.

En relación con los factores de riesgo, no se encontró asociación estadísticamente significativa en el presente estudio, a pesar de que la población estaba expuesta a diferentes factores, coincidente con lo reportado en países como Perú y México $(14,23)$. En el presente estudio se encontraron factores de riesgo relacionados con leptospirosis, como el trabajar o realizar la práctica clínica veterinaria; falta de uso de elementos de bioseguridad, como guantes y tapabocas; y presentar accidentes de trabajo. Estos resultados son similares a los reportados en Chile, donde se identificaron como factores de riesgo desarrollar labores agrícolas, contacto con agua de acequias, limpieza de lugares cerrados donde se había detectado la presencia de roedores y la falta de protección personal en labores de alto riesgo, como el uso de 
botas de goma para actividades de regadío (26-29). En un estudio realizado en Turbo, Colombia, se encontró como factor de riesgo y estadisticamente significativo el caminar descalzo dentro y fuera de la vivienda y tener animales no domésticos (22), datos que difieren con los resultados del presente estudio, donde se encontraron como factores protectores.

De acuerdo con los resultados obtenidos en este estudio, se puede observar que la Leptospirosis es una enfermedad de gran importancia y de elevada presentación, principalmente entre la poblacion que se encuentra constantemente en riesgo ocupacional al trabajar con animales. Es de vital importancia la continuación de estudios en el departamento con el fin de conocer la dinamica de la enfermedad, reconociendo el peligro que esta representa al ser una zoonosis-antropozoonosis, que requiere gran atención en cuanto al riesgo que representa para la salud pública.

Financiación: este proyecto fue financiado por el CIPADE - Centro de Investigaciones para el Desarrollo de la Universidad de Boyacá.

Conflicto de interés: Los autores declaran que no existe conflicto de intereses.

\section{Referencias}

1. Romero EC, Bernardo CC, Yasuda PH. Human leptospirosis: a twenty-nine-year serological study in Sao Paulo, Brazil. Rev Inst Med Trop Sao Paulo 2003; 45(5):245-8. DOI: org/10.1590/S0036-46652003000500002

2. Levett P. Leptospirosis: A forgotten zoonosis? Rev Clin Applied Immunol 2004; 4: 435-48.

3. Zunino E, Pizarro R. Leptospirosis. Puesta al día. Rev Chilena Infectol 2007; 24(3): 220-6. DOI: org/10.4067/S071610182007000300008

4. Adler B, Lo M, Seemann T, Murray G. Pathogenesis of leptospirosis: The influence of genomics. Vet Microbiol 2011; 153(1-2): 73-81. DOI: 10.1016/j.vetmic.2011.02.055. Epub 2011 Mar 5. Review.
5. Dabanch J. Zoonosis. Rev Chilena Infectol 2003; 20(Supl 1): 47-51. DOI: org/10.4067/S0716-10182003020100008

6. Alonso, B., Gómez de Haz, H., Pérez, B., Cruz de la Paz, R. Diagnóstico y tratamiento de la leptospirosis humana. Rev Cubana Med Gen Integr 2001; 17(1): 68-73.

7. Cediel, N., Villamil, L. Riesgo biológico ocupacional en la medicina veterinaria, área de intervención prioritaria. Revista de Salud Pública 2004; 6(1): 28-43.

8. World Health Organization. Enfermedades relacionadas por aguas, Leptopirosis [en línea]. Health Topics World Health Organization. [Ginebra, Suiza] Health Topics. 2013.

9. Instituto Nacional de Salud (INS). Protocolo de Vigilancia en Salud Pública, Leptospirosis. 2014. [Internet] [Consultado 26 Mar 2015] Disponible en: http://www.ins.gov.co/ tramites-y-servicios/examenes-de-inter\%C3\%A9s-en-salud-publica/Microbiologa/GUIA\%20PARA\%20LA\%20 VIGILANCIA\%20POR\%20LABORATORIO\%20DE\%20 LEPTOSPIRA.pdf

10. Ministério da Saúde, Secretaria de Vigilância em Saúde, Departamento de Vigilância das Doenças Transmissíveis; Leptospirose - Diagnóstico e Manejo Clínico. Brasilia DF, 2014. [Internet] [Consultado 10 de febrero 2015] Disponible en: http://www.dive.sc.gov.br/conteudos/publicacoes/leptospirose-diagnostico-manejo-clinico.pdf

11. Grupo Técnico Interinstitucional del Comité Nacional para la Vigilancia Epidemiológica (CoNaVE); Manual de Procedimientos Estandarizados para la Vigilancia Epidemiológica de la Leptospirosis. 2012.

12. Boletín Integrado de Vigilancia, Secretaria de Promoción y Programas sanitarios $N^{\circ} 240$ - SE 53. 2014.

13. Martínez P, Ortega D, Salinas K. Evolución de la leptospirosis según el Sistema de Vigilancia Epidemiológica Nacional, Chile 2003-2009. Rev Chilena Infectol. 2012; 29(6): 648-654. DOI: org/10.4067/S0716-10182012000700010

14. Dammert N, Noé N, Falcón N, Lopera L, Rodríguez M. Exposición a Leptospira sp. en estudiantes de medicina veterinaria al inicio y término de la carrera. Rev Inv Vet Perú. 2009; 20(1): 114-119

15. Instituto Nacional de Salud (INS) Dirección de Vigilancia y Análisis del Riesgo en Salud Pública, Boletín epidemiológico semanal; Semana epidemiológica número 16, 2015.

16. Soledad Bermúdez C, Martín Pulido M, Roy Andrade B. Seroprevalencia de Leptospira spp en caninos y humanos de tres barrios de Tunja, Colombia. Rev. MVZ Córdoba. 2010; 15(3): 2185-2193.

17. Ferro B, Rodríguez A, Pérez M, Travi B. Seroprevalencia de infección por Leptospira en habitantes de barrios periféricos de Cali. Biomédica 2006; 26: 250-7.

18. Góngora A, Parra JL, Aponte LH, Gómez LA. Seroprevalencia de Leptospira spp. En grupos de población de Villavicencio, Colombia. Rev Salud Pública 2008; 10(2): 269-78. 
19. Adriana María pedraza, Erika Esperanza Salamanca, Román Yesid Ramírez, Juan Manuel Ospina, Martín Orlando pulido. Seroprevalencia de anticuerpos anti-Leptospira en trabajadores de plantas de sacrificio animal en Boyacá, Colombia. Infectio 2012; 16(1): 31-36

20. Parasuraman B, Pichai K, Karumana K. Study on the Prevalence of Leptospirosis among Fever Cases Reported from Private Clinics in the Urban areas of Villupuram District, Tamil Nadu, India. Osong Public Health Res Perspect. 2014; 5(1): 54-67. DOI: 10.1016/j.phrp.2014.01.003

21. A.A. Noor Rafizah, B.D. Aziah, Y.N. Azwany, M. Kamarul Imran, A. Mohamed Rusli, S. Mohd Nazri a, A. Mohd Nikman b, I. Nabilah c, H. Siti Asma' c, W.M. Zahiruddin, I. Zaliha. A hospital-based study on seroprevalence of leptospirosis among febrile cases in northeastern Malaysia. International Journal of Infectious Diseases. 2013; 17(6): e394-e397. DOI: org/10.1016/j.ijid.2012.12.012

22. Yusti D, Arboleda M, Agudelo-Flórez P. Factores de riesgo sociales y ambientales relacionados con casos de Leptospirosis de manejo ambulatorio y hospitalario, Turbo, Colombia. Biomédica 2013; 33: 117-29. DOI: org/10.7705/biomedica. v33i0.1457

23. Vado-Solís IA, Cárdenas-Marrufo MF, Pérez-Osorio CE, Jiménez-Delgadillo BM, Arias-León JJ, Peniche-Lara F. Leptospirosis: una enfermedad ocupacional en trabajadores que conviven con bovinos seropositivos en el estado de Yucatán. Ciencias Humanismo Salud 2014; 1(1): 4-13.

24. 24. Cristancho - Torres DS, Benítez - Cabrera KA, Góngora - Orjuela A. Conocimientos sobre leptospirosis en estudiantes de veterinaria y seropositividad, Villavicencio, 2011.ORINOQUIA 2012; 16(2): 118-124.

25. Vado-Solis I, Cárdenas-Marrufo MF, Jiménez-Delgadillo B, Alzina-López A, Laviada-Molina H,Suárez-Solis V, et al. Clinical-epidemiological study of leptospirosis in humans and reservoirs in Yucatán, México. Rev Inst Med Trop Sao Paulo 2002; 44(6):335-40.

26. Perret C, Abarca K, Dabanch J, Solari V, García P, Carrasco S, Olivares R, Avalos P. Prevalencia y presencia de factores de riesgo de leptospirosis en una población de riesgo de la Región Metropolitana. Rev Méd Chile 2005; 133:426-431.

27. Hernádez P, Quintero G. Etiología bateriana de infecciones oculares externas. NOVA. 2003; 1(1); 57-64.

28. Páez, L. C. C., et al. Comparación del cultivo celular de HeLa y HEp-2: Perspectivas de estudios con Chlamydia trachomatis. 2015; Nova 13(23)

29. Flórez, R. A. N. Avances y perspectivas en Síndrome de Asperger. 2014; Nova, 12(21). 